\title{
Yiqi Huayu Jiedu Decoction Inhibits the Invasion and Metastasis of Gastric Cancer Cells through TGF- $\beta /$ Smad Pathway
}

\author{
Ting-Ting Wu, ${ }^{1,2}$ Jun Lu, ${ }^{1,2}$ Pei-Qiu Zheng, ${ }^{1}$ Shen-Lin Liu, ${ }^{1}$ Jian Wu, ${ }^{1,2}$ Wei Sun, \\ Qing-Min Sun, ${ }^{1}$ Nai-Xia Ma, ${ }^{1,2}$ Xue-Lian Ding, ${ }^{1,2}$ Min Chen, ${ }^{1}$ and Xi Zou ${ }^{1}$ \\ ${ }^{1}$ The Affiliated Hospital of Nanjing University of TCM, Jiangsu Province Hospital of TCM, Nanjing 210029, China \\ ${ }^{2}$ No. 1 Clinical Medical College, Nanjing University of Chinese Medicine, Nanjing, Jiangsu 210023, China \\ Correspondence should be addressed to Xi Zou; zouxidr@126.com
}

Received 30 November 2016; Accepted 9 March 2017; Published 30 April 2017

Academic Editor: Darren R. Williams

Copyright (c) 2017 Ting-Ting Wu et al. This is an open access article distributed under the Creative Commons Attribution License, which permits unrestricted use, distribution, and reproduction in any medium, provided the original work is properly cited.

Background. Yiqi Huayu Jiedu Decoction (YHJD) can obviously improve the quality of life of those patients with gastric cancer and prolong their survival. Methods. In vitro experiments, we observe YHJD's effect on the cells' proliferation by MTT assay. Cell adhesion assay, wound-healing assay, and Transwell invasion assay serve to detect its influence on cells' adhesion, migration, and invasion, respectively. Inhibitor $(10 \mu \mathrm{M} / \mathrm{L}$ of SB431542) and activator $(10 \mathrm{ng} / \mathrm{mL}$ of TGF- $\beta)$ of TGF- $\beta /$ Smad pathway were used to estimate whether YHJD's impact on the biological behavior of gastric cancer cells was related to TGF- $\beta /$ Smad pathway. In in vivo studies, YHJD was administered to the nude mice transplanted with gastric cancer to observe its effect on the tumor. Western blotting and immunohistochemical assay were used to test relevant cytokines of TGF- $\beta /$ Smad pathway and epithelial-mesenchymal transition (EMT) in MGC-803 cells and the tumor bearing nude mice. Results. YHJD inhibited proliferation, adhesion, migration, and invasion of MGC-803 gastric cancer cells in vitro. In in vivo studies, YHJD reduced the volume of the transplanted tumors. It also enhanced the expression of E-cadherin and decreased the levels of N-cadherin, TGF- $\beta$, Snail, and Slug in both MGC-803 cells and the transplanted tumor by western blot assay. The immunohistochemical assay revealed that YHJD raised E-cadherin in the tumors of the mice; on the contrary, the expression of N-cadherin, Twist, vimentin, TGF- $\beta$ R I, p-Smad2, p-Smad3, Snail, and Slug reduced. Conclusion. YHJD can effectively inhibit the invasion and metastasis of gastric cancer cells. The mechanism may be related to TGF- $\beta /$ Smad pathway.

\section{Introduction}

Gastric cancer is one of the most common malignant tumors in China, which is identified as the leading cause of cancer death [1]. A survey demonstrates that the primary reason for the death of patients with gastric cancer is recurrence and distant metastasis of the tumor. The relapse rates of patients with gastric cancer who have undergone surgery are 50\% within one year and $70 \%$ within two years, while $80 \%$ of the patients were found to exhibit local or distant metastasis in autopsy after death $[2,3]$. Therefore, drugs against the growth and metastasis of gastric cancer are urgently required.

Natural medicine is an important source of antitumor chemotherapy, and to find effective ingredients from natural medicine is one of the important strategies of antitumor drug development today. Yiqi Huayu Jiedu Decoction (YHJD) is comprised of Shenghuangqi (Radix Astragali), Dangshen (Radix et Rhizoma Salviae Miltiorrhizae), Sanleng (Rhizoma Sparganii), Ezhu (Rhizoma Curcumae), Danshen (Radix et Rhizoma Salviae Miltiorrhizae), Yinchen (Herba Artemisiae Scopariae), Banzhilian (Herba Scutellariae Barbatae), Dijincao (Herba Euphorbiae Humifusae), and Baqia (Rhizoma Smilacis Chinae), which is a summary of 40 years of clinical experience of the nationally renowned TCM (traditional Chinese medicine) doctor Professor Liu Shenlin. Clinical study on large samples has shown that YHJD can obviously improve the quality of life of patients and prolong their survival, but it is rare to see researches into its underlying molecular mechanisms. However, formulae have their specific and complicated effects through a variety of pathways and targets due to 
multiple compositions and complicated metabolic processes. Thus, the underlying molecular mechanisms associated with the therapeutic potential for gastric cancer remain unknown.

The first step of tumor metastasis is the weakening of the intercellular adhesive ability, the increase of the intercellular invasive ability, and finally falling off of tumor cells from the primary lesion. Epithelial-mesenchymal transition (EMT) plays a crucial role during cancer invasion and metastasis $[4,5]$. The TGF- $\beta /$ Smad pathway which is activated by transforming growth factor- $\beta$ (TGF- $\beta$ ) is the main mechanism of EMT $[6,7]$. In addition, studies have found that an increased expression of TGF- $\beta$ is found in various human cancers, including gastric cancer $[8,9]$. Thus, targeting TGF- $\beta$ can be considered as a potential strategy in the prevention and treatment of gastric cancer.

This study aims to reveal that YHJD can inhibit the invasion and metastasis of gastric cancer through TGF$\beta /$ Smad pathway in vivo and in vitro.

\section{Materials and Methods}

2.1. Cells and Animals. Human gastric cancer cell line MGC803 was under routine culture at the Centre Laboratory of Jiangsu Provincial Hospital of TCM. It was cultured in RPMI1640 medium (HyClone, Thermo Scientific, USA) supplemented with $10 \%$ fetal calf serum (Evergreen Company, Hangzhou, China). The cells were incubated at $37^{\circ} \mathrm{C}, 5 \% \mathrm{CO}_{2}$, and saturated humidity atmosphere.

$24 \mathrm{BALB} / \mathrm{c}$ mice (number 201605969) were purchased from Charles River, Beijing, China. The mice were half male and half female, 4 weeks old, weighing 18-22 g. They were fed in SPF environment with regular indoor ultraviolet radiation. Their cage, litter, feeds, and water were strictly sterilized.

2.2. Yiqi Huayu Jiedu Decoction (YHJD). All the medical herbs were sourced from Jiangsu Province Hospital of TCM. Herbs (165 g), including $30 \mathrm{~g}$ Shenghuangqi, $30 \mathrm{~g}$ Dangshen, $15 \mathrm{~g}$ Sanleng, $15 \mathrm{~g}$ Ezhu, $15 \mathrm{~g}$ Danshen, $15 \mathrm{~g}$ Yinchen, $15 \mathrm{~g}$ Banzhilian, $15 \mathrm{~g}$ Dijincao, and $15 \mathrm{~g}$ Baqia, were boiled with $1650 \mathrm{~mL}$ of distilled water, refluxed, and extracted. $4 \mathrm{~g} / \mathrm{mL}$ herb water extract was obtained from the mixed decoctions and then filtered through a $0.2 \mu \mathrm{m}$ filter. The extract was stored at $-20^{\circ} \mathrm{C}$ until use.

2.3. MTT Assay. Cells in the logarithm phase were seeded in 96-well plates at the density of $6 \times 10^{3} /$ well.

When the cells were adherent to the walls, the experimental groups were divided into control group, TGF- $\beta$ group, TGF- $\beta$ + YHJD group, SB431542 group, SB431542 + YHJD group, and YHJD group, respectively. The control group were add equal amount of complete medium: TGF- $\beta$ at $10 \mathrm{ng} / \mathrm{mL}$ (Peprotech Bio Co., Ltd., China), SB431542 at $10 \mu \mathrm{M}$ (SigmaAldrich, St. Louis, MI, USA), and YHJD at $2 \mathrm{mg} / \mathrm{mL}$. Then, the cells were further incubated at $37^{\circ} \mathrm{C}$ with $5 \% \mathrm{CO}_{2}$ for $24 \mathrm{~h}, 48 \mathrm{~h}$, and $72 \mathrm{~h}$, respectively, followed by adding $20 \mu \mathrm{L}$ of MTT (5 g/L) which was purchased from Sigma-Aldrich (St. Louis, MI, USA) and incubating for $4 \mathrm{~h}$. The supernatant was removed, and then $150 \mu \mathrm{L}$ of dimethylsulfoxide (DMSO) was added. Absorbance under $490 \mathrm{~nm}$ was detected to calculate the inhibition rate. Inhibition rate $(\%$ of control $)=(1-$ absorbance of test sample/absorbance of control) $\times 100 \%$. The test was repeated three times.

2.4. Cell Adhesion Assay. 96-well plates were coated with $70 \mu \mathrm{g} / \mathrm{mL}$ FN (BD Biosciences, San Diego, CA) with $30 \mu \mathrm{L}$ per well, dried on a clean bench, and prepared at $4^{\circ} \mathrm{C}$. BGC803 gastric cancer cells were seeded in culture dishes at $1 \times$ $10^{5} / \mathrm{mL}$. Then, drugs were administered according to their groups: control group, TGF- $\beta$ group, TGF- $\beta+$ YHJD group, SB431542 group, SB431542 + YHJD group, and YHJD group. After $24 \mathrm{~h}$, cells were digested, washed, and resuspended with a drug-containing medium and then seeded in 96well plates at $1 \times 10^{5}$ per well. The original drug-containing medium was replaced by complete medium every $30 \mathrm{~min}$. Until $120 \mathrm{~min}, 20 \mu \mathrm{L}$ of MTT $(5 \mathrm{~g} / \mathrm{L})$ was added to each well and incubated for $4 \mathrm{~h}$. The supernatant was removed and $150 \mu \mathrm{L}$ of dimethylsulfoxide was added to each well. Absorbance under $490 \mathrm{~nm}$ was detected to calculate the inhibition rate.

2.5. Wound-Healing Assay. BGC-803 gastric cancer cells were seeded in 24 -well plates at $1 \times 10^{5} / \mathrm{mL}$. Once $100 \%$ confluence was observed, the cell monolayer was scratched with a pipette tip after washing with phosphate buffer saline (PBS) twice. The relative distance between wound boundaries was recorded by microscopic scanning. Then, drugs were administered according to their groups: control group, TGF$\beta$ group, TGF- $\beta+$ YHJD group, SB431542 group, SB431542 + YHJD group, and YHJD group. After $24 \mathrm{~h}$ culture, the widths of the scratches were photographed for analysis. Wound closure $(\%)=$ healing boundary/wound boundary.

2.6. Transwell Assay. BGC-803 cells were seeded in culture dishes at $1 \times 10^{5} / \mathrm{mL}$ and starved in serum-free RPMI1640 medium for $24 \mathrm{~h}$. Afterwards, they were digested and inoculated at $6 \times 10^{5} / 100 \mu \mathrm{L}$ in small chambers coated with Matrigel (Millipore, Germany). The chambers were placed in 24-well plates, while $1 \%$ FBS was added to the upper chambers and $10 \%$ FBS was added to the lower chambers. Drugs were administered according to their groups at the same time: control group, TGF- $\beta$ group, TGF- $\beta+$ YHJD group, SB431542 group, SB431542 + YHJD group, and YHJD group. After $24 \mathrm{~h}$, nonpenetrative cells on the top chamber were wiped away, and the migrating cells were fixed and stained with $0.1 \%$ crystal violet. The basement membrane was removed to get five photos of the cells of each group at random selection of microscope fields.

2.7. Animal Studies. Gastric cancer MGC-803 cells in the logarithmic growth phase were resuspended and digested at $2.5 \times 10^{7} / \mathrm{mL}$. Each mouse was inoculated with $0.2 \mathrm{~mL}$ of cell suspension in the armpit. After two weeks, the diameter of the induration grew up to $10 \mathrm{~mm}$. And a successful model was built. The 24 mice were randomly divided into four groups according to the mode of administration: control group (NS $0.2 \mathrm{~mL} / 10 \mathrm{~g}$ via gavage), 5 -FU group (5-FU $0.1 \mathrm{~mL} / 10 \mathrm{~g}$, $2.5 \mathrm{mg} / \mathrm{kg}$ via intraperitoneal injection), high-dose group (YHJD $4 \mathrm{~g} / \mathrm{mL}, 0.2 \mathrm{~mL} / 10 \mathrm{~g}$ via gavage), and low-dose group 
(YHJD $2 \mathrm{~g} / \mathrm{mL}, 0.2 \mathrm{~mL} / 10 \mathrm{~g}$ via gavage). They were medicated for 14 days.

2.8. Observation on Antitumor Effect. Daily diet, defecation, urination, active status, and tumor growth of the mice were observed. After administration, the short- and long-axis diameters of the tumor were measured every other day. The volume of the tumor was calculated according to Steel formula $(\mathrm{V})=\left(\right.$ long diameter $\times$ short diameter $\left.{ }^{2}\right) / 2\left(\mathrm{~cm}^{3}\right)$. Volume inhibition rate $(\%)=\left(1-\mathrm{V}_{\text {experimental group }} / \mathrm{V}_{\text {control group }}\right) \times$ $100 \%$. The mice were sacrificed on the 14 th day after administration of drugs.

2.9. Immunohistochemistry Assay. Paraformaldehyde-fixed tumor tissue sections ( $4 \mathrm{~mm}$ thick) were mounted on slides, dewaxed, and hydrated. Slides were boiled in $10 \mathrm{mM}$ sodium citrate buffer ( $\mathrm{pH}$ 6) for $2 \mathrm{~min}$ and cooled on bench top for $30 \mathrm{~min}$, followed by incubation in 3\% hydrogen peroxide for $15 \mathrm{~min}$ and blocking with normal goat serum for $30 \mathrm{~min}$. Sections were incubated with primary antibodies (E-cadherin, $\mathrm{N}$-cadherin, vimentin, Snail, Slug, TGF- $\beta$ R I, p-Smad2, and p-Smad3) and then washed with PBST buffer and incubated with HRP conjugated anti-rabbit or anti-mouse IgG (Boshide Biological Technology Co., Ltd., Wuhan, China). Localization of peroxidase conjugates was revealed using diaminobenzidine tetrahydrochloride solution and chromogen and hematoxylin for counterstaining. Protein expression was observed under an optical microscope. Image-Pro was adopted to analyze each picture to get their integrated optical density (IOD) and area. Mean density (IOD/area) was used to analyze the expression.

2.10. Western Blot Analysis. BGC-803 cells were administered with drugs for $24 \mathrm{~h}$ according to their groups: control group, TGF- $\beta$ group, TGF- $\beta$ + YHJD group, SB431542 group, SB431542 + YHJD group, and YHJD group. Transplanted tumors in each group (control group, 5-FU group, highdose group, and Low-dose group) were peeled. The protein lysates from cultured cells and tumor were separated on $10 \%$ sodium dodecylsulfate polyacrylamide gel electrophoresis (SDS-PAGE) and transferred to PVDF membranes (Millipore, Billerica, MA, USA). Membranes were blocked with skim milk, followed by incubation with the primary antibodies against E-cadherin, N-cadherin, vimentin, TGF-b, Smad2, Smad3, Snail, Slug, and Twist (Twist was purchased from Abcam, USA, and others were from Cell Signaling Technology, Beverly, MA). Incubation was carried out with horseradish peroxidase-conjugated secondary antibody (Cell Signaling Technology, MA, USA). Signals were observed under the Image Studio version 3.1.4 system.

2.11. Statistical Analysis. All data were expressed as means \pm standard deviation (SD). One-way ANOVA was used to determine statistically significant differences between groups. Scheffe's test (SPSS 18.0, SPSS Inc., Chicago, IL) was used to correct for multiple comparisons when statistical significance was identified in ANOVA. Statistical significance was set at $P<0.05$.

\section{Results}

3.1. YHJD Time-Dependently Inhibited the Proliferation of Gastric Cancer Cell MGC-803. Cells of the control group was found to be pebble-like under the inverted microscope, and connection between the cells was closer, like that among epithelial cells. Cells of the TGF- $\beta$ group were significantly elongated, like interstitial cells. Compared with the control group, cells of SB431542 group and YHJD group were polygonal, and YHJD time-dependently inhibited the proliferation of MGC-803 cells $(P<0.01)$; its inhibition rate reached $49.65 \pm 0.62 \%, 50.94 \pm 2.56 \%$, and $61.49 \pm 1.05 \%$ at $24 \mathrm{~h}, 48 \mathrm{~h}$, and $72 \mathrm{~h}$, respectively. The results also showed that, compared with SB431542 group, YHJD more strongly inhibited cells from proliferation $(P<0.01)$, as shown in Figures $1(\mathrm{a})$ and $1(b)$.

3.2. YHJD Prohibited Adhesion, Migration, and Invasion of MGC-803 Cells. Compared with the control group, TGF$\beta$ enhanced adhesion and migration of the cells $(P<$ 0.01); both SB431542 and YHJD suppressed cells' adhesion, migration, and invasion $(P<0.01)$; compared with SB431542 group, YHJD group and SB431542 + YHJD group had a stronger effect on the inhibition of adhesion and invasion of cells $(P<0.01)$, as shown in Figures $1(\mathrm{c})-1(\mathrm{~g})$.

3.3. YHJD Inhibited the Transplanted Tumor Growth in the Mice. The mental state of the animals, intake of food and water, urination, defecation, and other activities were not significantly different between groups. The mice were randomly divided into control group, 5-FU group, high-dose group, and low-dose group. Compared with the control group, the weights of the mice in each group were not significantly different during the experiment $(P>0.05)$. After 5 days of administration, the average tumor volume in the mice of the high-dose group and low-dose group was smaller than that of the control group $(P<0.05)$. At the end of the experiment, compared with the control group, the high-dose group and 5FU group were found to significantly inhibit the tumor from growing $(P<0.01)$, as shown in Figures $2(\mathrm{a})$ and $2(\mathrm{~b})$.

3.4. YHJD Upregulated the Expression of E-Cadherin through Inhibition of TGF- $\beta /$ Smad Pathway. Immunohistochemistry showed that, compared with the control group, E-cadherin level increased $(P<0.05)$ in the high-dose group, low-dose group, and 5-FU group, while levels of $\mathrm{N}$-cadherin, Twist, vimentin, TGF- $\beta$ R I, p-Smad2, p-Smad3, Snail, and Slug declined $(P<0.05)$, as shown in Figures 3(a) $-3(\mathrm{j})$.

\subsection{TGF- $\beta$ Inhibited the Expression of E-Cadherin through} Activation of TGF- $\beta /$ Smad Pathway, Which Can Be Abolished by YHJD. Western blot showed that, in the cell assay, compared with the control group, E-cadherin of TGF- $\beta$ group declined, while the level of N-cadherin, vimentin, TGF- $\beta$, and Snail increased. Compared with the control group, Ncadherin, vimentin, and Snail of SB431542 group declined. Compared with the control group and TGF- $\beta$ group, Ecadherin of YHJD group and SB431542 + YHJD group increased, while the level of $\mathrm{N}$-cadherin, vimentin, TGF- $\beta$, 


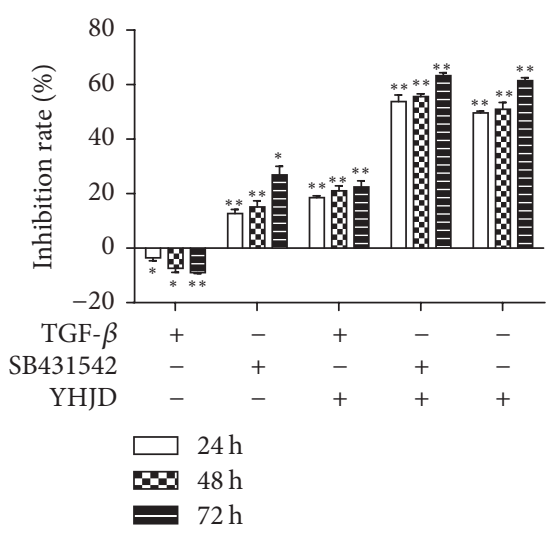

(a)
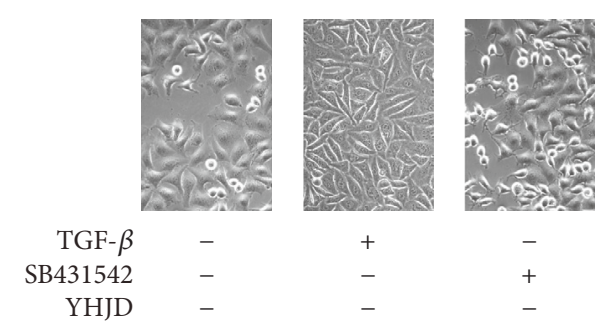

(b)

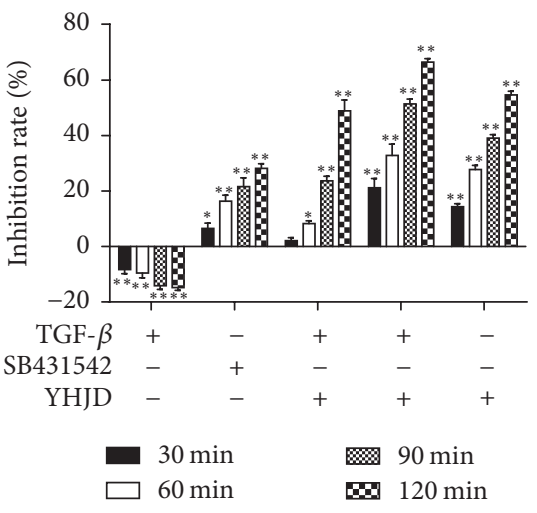

(c)
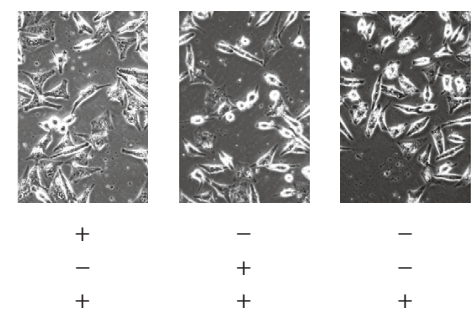
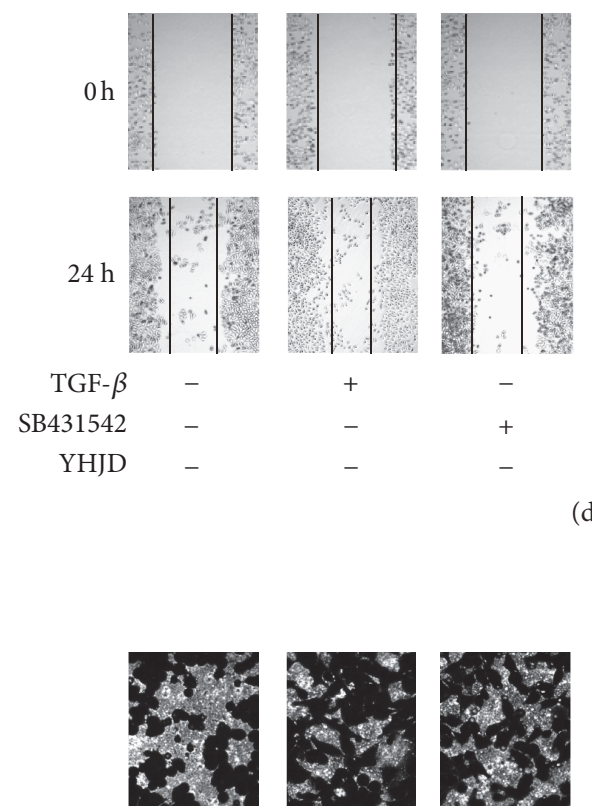

TGF- $\beta$

SB431542

YHJD
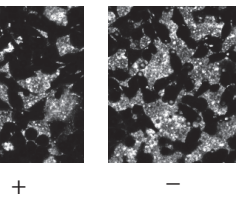

$+$

$-$
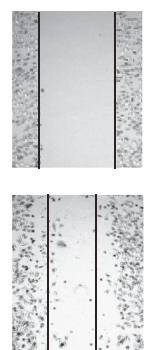

(d)
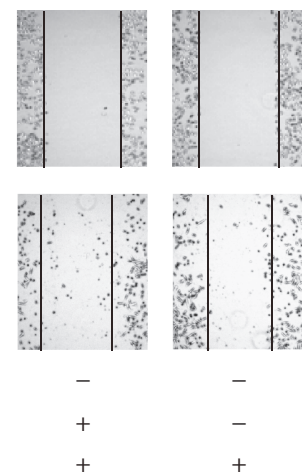

$+$

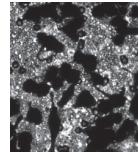

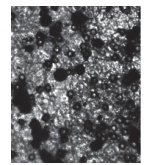

$+$

$-$

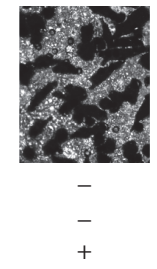

(f)

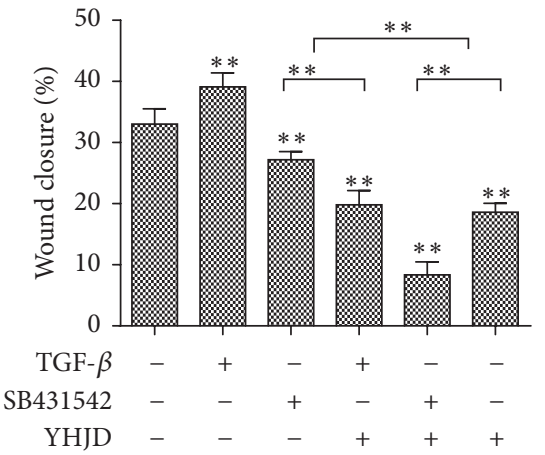

(e)

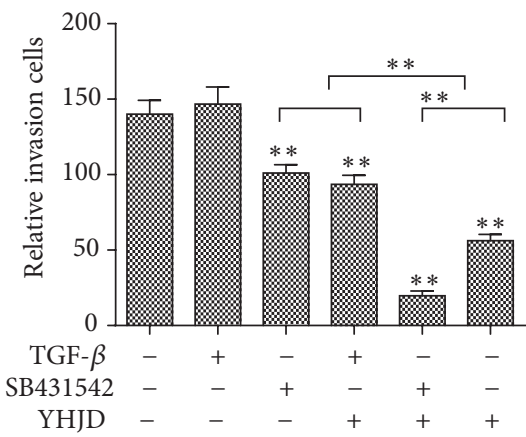

(g)

FIGURE 1: YHJD inhibited gastric cancer MGC-803 cells' proliferation, adhesion, migration, and invasion. (a) TGF- $\beta$ (10 ng/mL), TGF- $\beta / \mathrm{Smad}$ $(10 \mu \mathrm{M} / \mathrm{L}$ SB431542), and YHJD $(2 \mathrm{mg} / \mathrm{mL})$ were administered to cells and cultivated for $24 \mathrm{~h}, 48 \mathrm{~h}$, and $72 \mathrm{~h}$, and the inhibition rate (\%) was determined using MTT assays and was expressed as (1 - absorbance of test sample/absorbance of control) $\times 100 \%$. (b) YHJD inhibited proliferation of gastric cancer cells and affected cells' morphology. (c) Cell adhesion assay was used to detect MGC-803 cells' adhesion for GC cells. The bar chart was the inhibition rate of cell adhesion of each group at different periods (30,60, 90, and 120 min). (d) Wound assay detected migration of different groups for MGC-803 cells, which were photographed $24 \mathrm{~h}$ after wounding (100x) to record their migration. (e) The bar chart shows wound-healing percentage of gastric cancer MGC- 803 cells $24 \mathrm{~h}$ after being wounded. (f) Transwell assay detected the effects of each group's invasion effect on MGC-803 cells. Light microscopic images of different concentrations (400x) were taken. (g) The bar chart shows the number of MGC-803 cells that migrated across $8 \mu \mathrm{m}$ diameter pores to the lower chamber. Data are expressed as the mean \pm SD of three experiments $\left({ }^{*} P<0.05\right.$ compared with control group, ${ }^{* *} P<0.01$ compared with control group). 


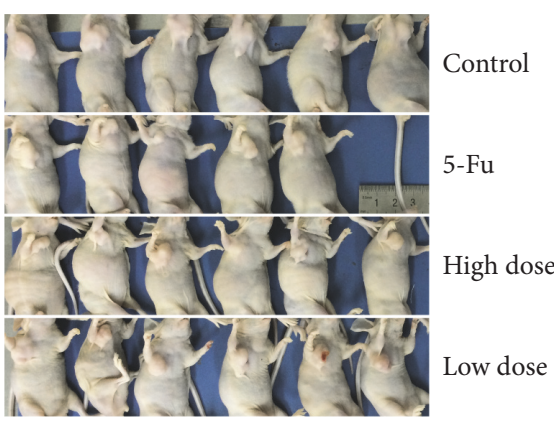

(a)

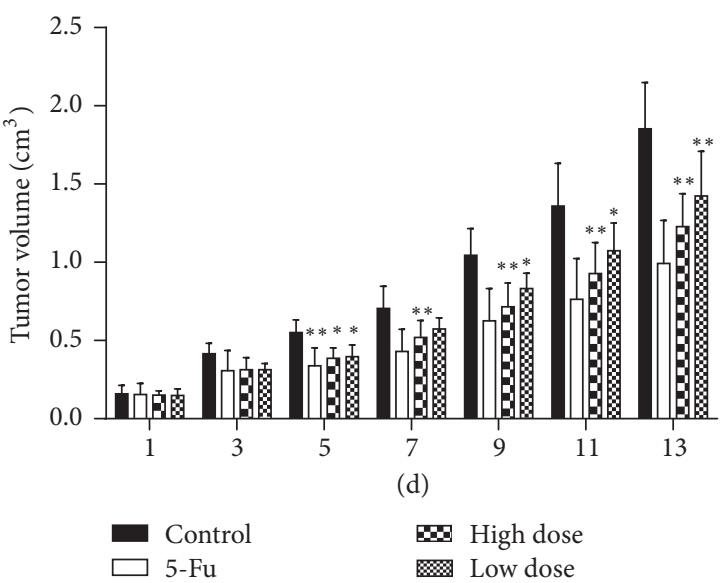

(b)

FIGURE 2: YHJD inhibited the growth of the transplanted tumor. (a) $5 \times 10^{6}$ MGC-803 cells were subcutaneously implanted in the mice. Two weeks after the tumors completely developed, they were divided into control group, 5-FU group, high-dose group, and low-dose group. The tumor was peeled out 14 days after administration. (b). The bar chart shows the change in metastatic tumor volume over medication time. Data are expressed as the mean $\pm \mathrm{SD}$ of 5 or 6 mice $\left({ }^{*} P<0.05\right.$ compared with control group, ${ }^{* *} P<0.01$ compared with control group).

and Snail declined. No significant difference in each factor's expression was found between YHJD group and SB431542 + YHJD group. No significant difference in Smad2, Smad3, Slug, and Twist expression was found between groups, as shown in Figures 4(a) and 4(b). In the transplanted tumor, compared with the control group, E-cadherin was upregulated in the high-dose group, low-dose group, and 5FU group, while the level of N-cadherin, vimentin, TGF- $\beta$, Snail, and Slug went downwards. No significant difference in Smad2, Smad3, Twist, and vimentin was found between groups, as shown in Figures 4(c) and 4(d).

\section{Discussion}

The recurrence and distant metastasis of gastric cancer, as one of the world's top 10 malignant tumors, have become a great threat to human health and a leading cause of mortality, and thus effective strategies are required. Traditional Chinese medicine has absolutely a big advantage in the treatment of various intractable diseases. At present, it is attracting more and more attention because it is multitargeted and treats both the tip and root of an illness, reduces toxins at the same time, and enhances efficacy, especially in the treatment of cancer. The Oncology Department of Jiangsu Provincial Hospital of TCM adopted an important treatment principle of replenishing qi, resolving stasis, and removing toxins for recurrence and metastasis of gastric cancer in long-term clinical practice. As an adjuvant therapy for gastric cancer patients after surgery, YHJD can improve the condition of qi deficiency and toxic stasis, enhance the effect of and patient's sensitivity to chemotherapy, and thus reduce the risk of recurrence and metastasis and improve the quality of life of patients. So far, it has gained a great therapeutic value. However, the mechanism of the effects of YHJD on the metastasis of gastric cancer has not been understood.
Recent studies have revealed that gastric carcinoma metastasis is closely related to epithelial-mesenchymal transition (EMT) $[10,11]$. Transforming growth factor- $\beta$ (TGF$\beta$ ) may play a critical role in EMT of malignant tumors belonging to epithelial origin $[7,12,13]$. Overexpression of TGF- $\beta$ activates TGF- $\beta /$ Smad pathway, and TGF- $\beta$ binds to TGF- $\beta$ receptors I and II to activate phosphorylated $\operatorname{Smad} 2 / 3$, thereby activating Smad 4 to transport TGF- $\beta /$ Smad signal into the cell nucleus to adjust EMT [14]. Therefore, in order to clarify the mechanism of TCM's inhibition of gastric cancer, the present study, based on TGF- $\beta /$ Smad pathway, observed YHJD's efficacy on gastric cancer. SB431542 has been shown to selectively block TGF- $\beta /$ Smad pathway through blocking the phosphorylation of Smad2 $[15,16]$. Since we use the gain and loss of function experiment to further verify the effect of YHJD, SB431542 has been shown to suppress the TGF$\beta$-induced EMT and proliferation of gastric cancer cells in humans. Therefore, we use TGF- $\beta$ as the activator of TGF$\beta /$ Smad pathway while SB431542 is used as the inhibitor. Cell experiments showed that, related to the control group, proliferation, adhesion, and migration of gastric cancer cells in TGF- $\beta$ group were improved, while proliferation, adhesion, and migration of gastric cancer cells in SB431542 group were reduced. The results indicated that activation of TGF$\beta /$ Smad pathway promoted the proliferation and metastasis of gastric cancer cells, consistent with literature reports. Compared with the control group, YHJD time-dependently inhibited the proliferation of gastric cancer cell MGC-803. YHJD can also prohibit adhesion, migration, and invasion of MGC-803 cells, which is in accordance with its clinical efficacy. Compared with TGF- $\beta$ group, TGF- $\beta+$ YHJD group can revert TGF- $\beta$ 's promotion effect on gastric cancer cells' proliferation, adhesion, and migration, indicating that this pathway might play an important role in YHJD's inhibition of gastric cancer development. Compared with SB431542 group, 


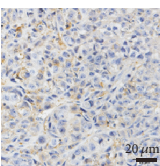

(I)

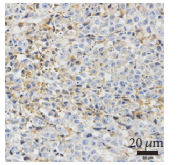

(III)

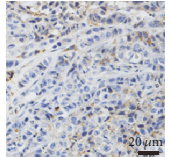

(II)

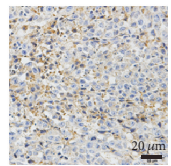

(IV)

(a)

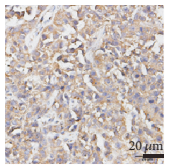

(I)

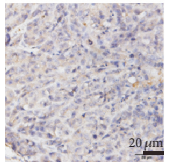

(III)

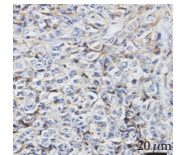

(I)

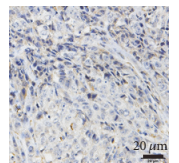

(III)

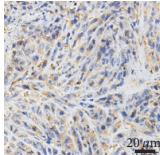

(II)

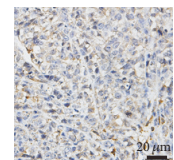

(IV)

(b)

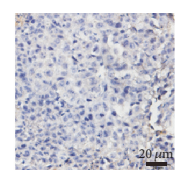

(II)

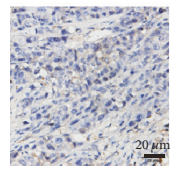

(III)

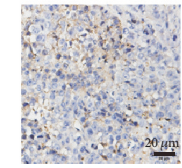

(IV)

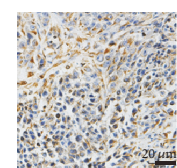

(I)

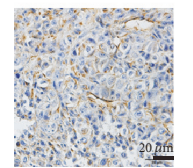

(III)

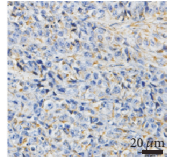

(II)

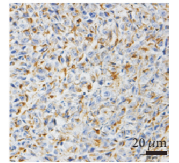

(IV)

(c)

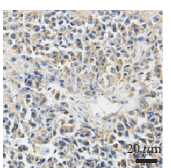

(I)

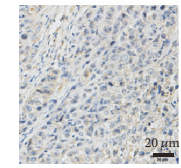

(III)

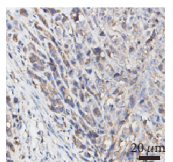

(I)

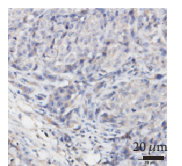

(III)

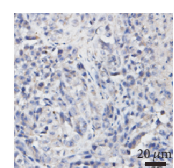

(II)

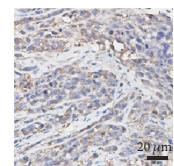

(IV)

(d)

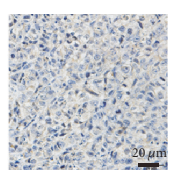

(II)

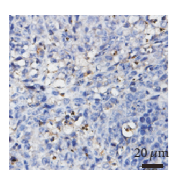

(I)

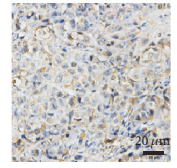

(IV)

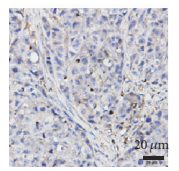

(III)

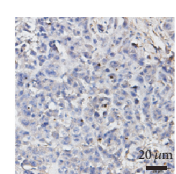

(II)

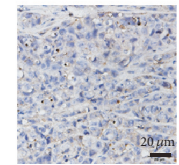

(IV) (e)

(f)

(g)

(h)
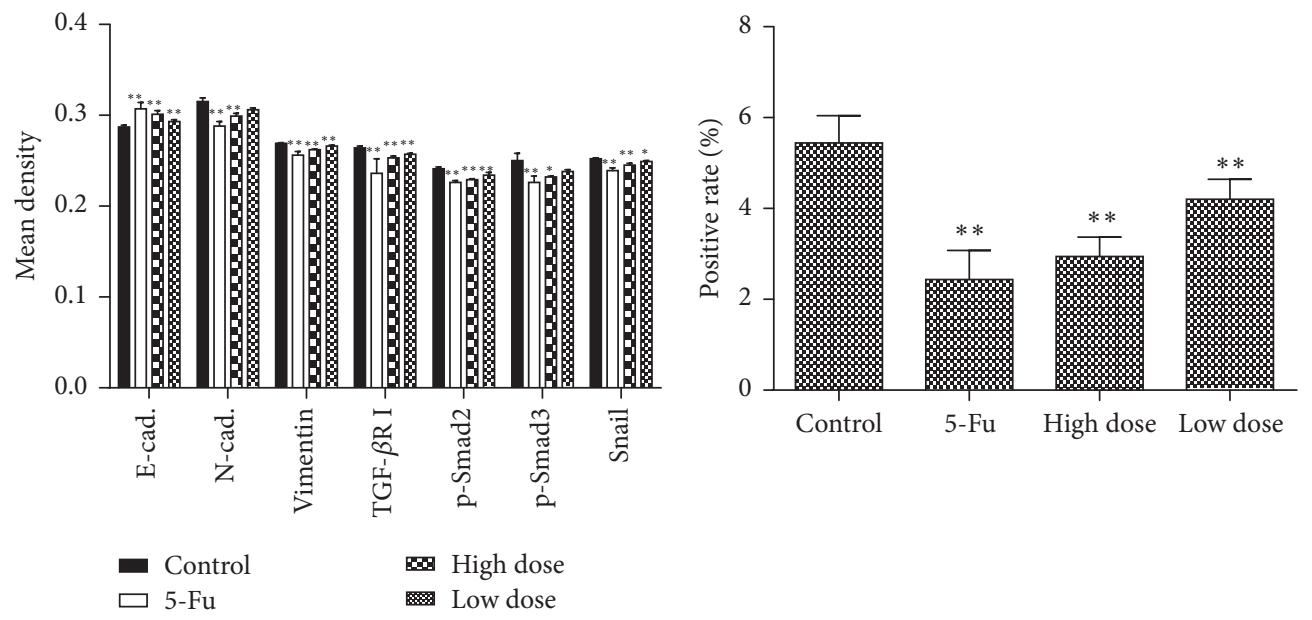

(i)

(j)

FIGURE 3: The levels of EMT and TGF- $\beta /$ Smad pathway related proteins in the transplanted tumor were determined by immunohistochemical assay. (a) E-cadherin. (b) N-cadherin. (c) Vimentin. (d) TGF- $\beta$ R I. (e) p-Smad2. (f) p-Smad3. (g) Snail. (h) Slug. Protein of (I) (control group), (II) (5-FU group), (III) (high-dose group), and (IV) (low-dose group) turned brown granular in tumor staining. Positive staining of E-cadherin, N-cadherin, and vimentin focused in the cytoplasm, a few in the envelope, brownish yellow granular. TGF- $\beta$ R I was mainly stained in the cell membrane. p-Smad2 and p-Smad3 in the cytoplasm, a few in the envelope. Snail in the cytoplasm, a few in the nucleus. Slug in the nucleus. (i) The bar chart indicates each molecule's mean density. (j) The bar chart indicates expression positive rate of Slug in metastatic tumor tissue sections. Data are expressed as the mean $\pm \mathrm{SD}$ of three experiments $\left({ }^{*} P<0.05\right.$ compared with control group, ${ }^{* *} P<0.01$ compared with control group).

YHJD group and SB431542 + YHJD group had a stronger ability to inhibit cell proliferation and metastasis, which may be related to YHJD's apoptosis induced function. That is, YHJD probably interfered with other mechanisms to inhibit gastric cancer as well, which was worthy of further studies. Our animal experiments illustrated that, compared with the control group, YHJD inhibited the tumor's growth in the mice, which was in accordance with clinical and experimental results.

Loss of epithelial polarity and gain of interstitial cells are characteristic marks of EMT $[17,18]$, including loss of expression and function of E-cadherin protein and other epithelial markers and overexpression of interstitial cell markers like Ncadherin and vimentin. Meanwhile, transcription factors like 


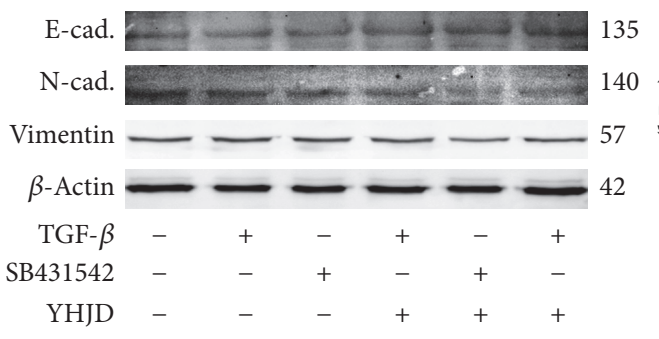

(a)

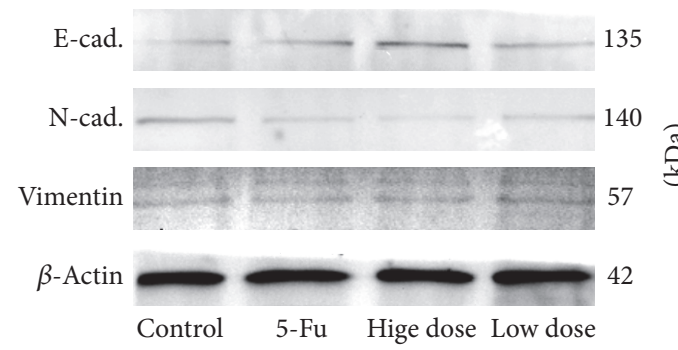

(c)

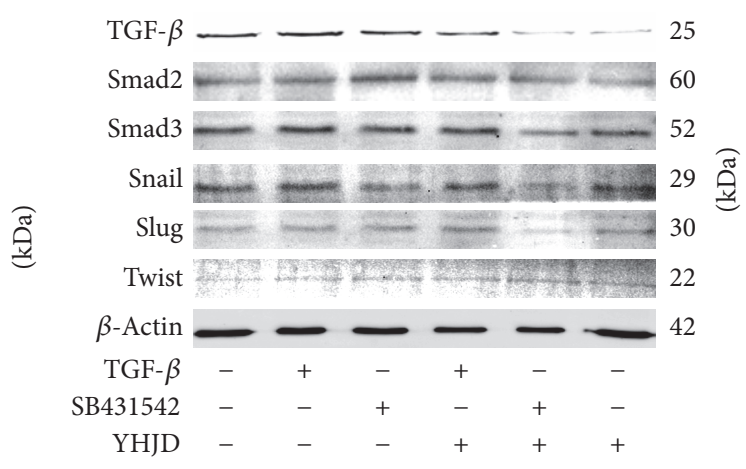

(b)

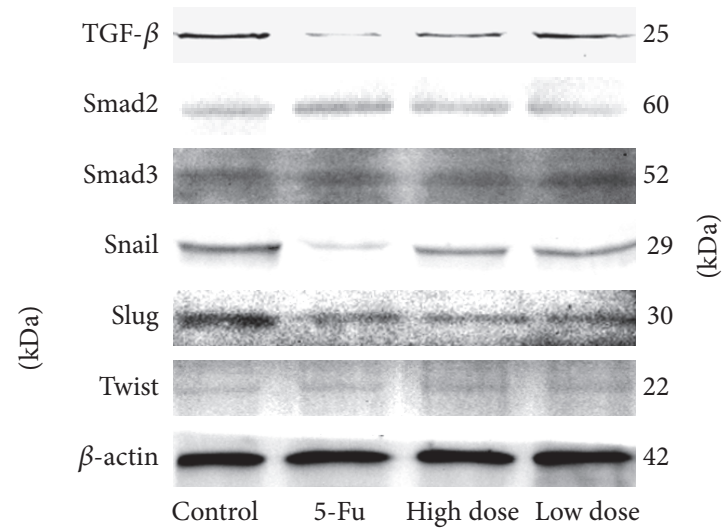

(d)

FIGURE 4: After MGC-803 was administered for $24 \mathrm{~h}$, the levels of (a) EMT (E-cadherin, N-cadherin, and vimentin) and (b) TGF- $\beta /$ Smad pathway (TGF- $\beta$, Smad2, Smad3, Twist, Snail, and Slug) related proteins were determined by western blot. (c-d) The protein expression in the transplanted tumor about EMT (E-cadherin, N-cadherin, and vimentin) and TGF- $\beta /$ Smad pathway (TGF- $\beta$, Smad2, Smad3, Snail, Slug, and Twist). $\beta$-Actin was taken as a loading control.

Snail, Slug, and Twist which negatively regulate E-cadherin were upregulated [19]. Therefore, local infiltration and distant metastasis of the tumor were promoted. TGF- $\beta /$ Smad pathway is the key to TGF- $\beta$-induced EMT. Inhibition of TGF$\beta /$ Smad pathway can reduce tumor invasion and metastasis [20]. In our cell experiments, western blot assay found that a high expression of TGF- $\beta$ was found in TGF- $\beta$ group, while TGF- $\beta+$ YHJD group can decrease its expression, indicating that this pathway might play an important role in YHJD's inhibition of gastric cancer development, which is in accordance with cell behavior experiments. YHJD can reduce the expression of TGF- $\beta$, prevent TGF- $\beta /$ Smad pathway from being activated, and thereby inhibit EMT by upregulating EMT-related phenotypes of E-cadherin and downregulating $\mathrm{N}$-cadherin, vimentin, Snail, and Slug. In animal experiments, immunohistochemistry and western blot assay found that YHJD reduced the expression of TGF- $\beta$, p-Smad2, and $\mathrm{p}$-Smad 3 and downregulated $\mathrm{N}$-cadherin and vimentin. This was in line with the results of cell experiments. Accordingly, we believe that YHJD may inhibit gastric cancer metastasis by inhibiting TGF- $\beta /$ Smad's activation. But there is still a possibility that YHJD inhibits EMT through other pathways or regulates other factors to activate TGF- $\beta /$ Smad pathway. This undoubtedly suggests that YHJD has multitarget antitumor activity, which is of great significance for the treatment of cancer.

Although there were important results in this study, there were also some limitations. For example, TGF- $\beta /$ Smad pathway is the only pathway we use to clarify YHJD's transfer resistance mechanism. More experiments, such as apoptosis related assays, are required to further identify the true active components and biological functions of this decoction.

\section{Conclusions}

Based on TGF- $\beta /$ Smad's signaling pathway, the present study explored the mechanism of YHJD in gastric cancer metastasis. It was found that YHJD could inhibit EMT through TGF$\beta /$ Smad pathway. Moreover, YHJD has multitarget antitumor activity, which is of scientific significance to the clinical practice and development of new drugs.

\section{Conflicts of Interest}

The authors declare that there are no conflicts of interest regarding the publication of this paper. 


\section{Authors' Contributions}

Ting-Ting Wu and Jun Lu contributed equally to this article. Pei-Qiu Zheng and Shen-Lin Liu analyzed and interpreted the data. Jian Wu, Wei Sun, and Qing-Min Sun performed the western blot examination. Nai-Xia Ma and Xue-Lian Ding performed the histological examination. Min Chen and Xi Zou were major contributors in writing the manuscript. All authors read and approved the final manuscript.

\section{Acknowledgments}

This work was supported by Jiangsu Provincial Special Program of Medical Science (BL2014100), by the National Natural Science Foundation of China (no. 81473605), and by the State Administration of Traditional Chinese Medicine of People's Republic of China (JDZX2015090).

\section{References}

[1] W. Chen, R. Zheng, P. D. Baade et al., "Cancer statistics in China, 2015," CA: A Cancer Journal for Clinicians, vol. 66, no. 2, pp. 115132, 2016.

[2] W. M. Kang, Q. B. Meng, J. C. Yu, Z. Q. Maand, and Z. T. Li, "Factors associated with early recurrence after curative surgery for gastric cancer," World Journal of Gastroenterology, vol. 21, no. 19, pp. 5934-5940, 2015.

[3] B. Wu, D. Wu, M. Wang, and G. Wang, "Recurrence in patients following curative resection of early gastric carcinoma," Journal of Surgical Oncology, vol. 98, no. 6, pp. 411-414, 2008.

[4] B. Smith and N. Bhowmick, "Role of EMT in metastasis and therapy resistance," Journal of Clinical Medicine, vol. 5, no. 2, artical 17, 2016.

[5] F. Guo, B. C. Parker Kerrigan, D. Yang et al., "Post-transcriptional regulatory network of epithelial-to-mesenchymal and mesenchymal-to-epithelial transitions," Journal of Hematology and Oncology, vol. 7, article 19, 2014.

[6] J. P. Thiery, H. Acloque, R. Y. J. Huang, and M. A. Nieto, "Epithelial-mesenchymal transitions in development and disease," Cell, vol. 139, no. 5, pp. 871-890, 2009.

[7] E. Zoni, G. van der Pluijm, P. C. Gray, and M. Kruithof-de Julio, "Epithelial plasticity in cancer: unmasking a microRNA network for TGF- $\beta$-, Notch-, and Wnt-mediated EMT," Journal of Oncology, vol. 2015, Article ID 198967, 13 pages, 2015.

[8] P. Mhawech-Fauceglia, J. Kesterson, D. Wang et al., "Expression and clinical significance of the transforming growth factor- $\beta$ signalling pathway in endometrial cancer," Histopathology, vol. 59, no. 1, pp. 63-72, 2011.

[9] M. D. Sides, R. C. Klingsberg, B. Shan et al., “The Epstein-Barr virus latent membrane protein 1 and transforming growth factor- $\beta 1$ synergistically induce epithelial-mesenchymal transition in lung epithelial cells," American Journal of Respiratory Cell and Molecular Biology, vol. 44, no. 6, pp. 852-862, 2011.

[10] J. Fuxe, T. Vincent, and A. G. de Herreros, “Transcriptional crosstalk between TGF $\beta$ and stem cell pathways in tumor cell invasion: role of EMT promoting Smad complexes," Cell Cycle, vol. 9, no. 12, pp. 2363-2374, 2010.

[11] J. Zhang, X. Tian, and J. Xing, "Signal transduction pathways of EMT induced by TGF- $\beta$, SHH, and WNT and their crosstalks," Journal of Clinical Medicine, vol. 5, no. 4, 2016.
[12] K. Miyazono, S. Ehata, and D. Koinuma, “Tumor-promoting functions of transforming growth factor- $\beta$ in progression of cancer," Upsala Journal of Medical Sciences, vol. 117, no. 2, pp. 143-152, 2012.

[13] A. M. Doerner and B. L. Zuraw, "TGF- $\beta 1$ induced epithelial to mesenchymal transition (EMT) in human bronchial epithelial cells is enhanced by IL-1 $\beta$ but not abrogated by corticosteroids," Respiratory Research, vol. 10, no. 1, p. 100, 2009.

[14] C. Andreolas, M. Kalogeropoulou, A. Voulgari, and A. Pintzas, "Fra-1 regulates vimentin during Ha-RAS-induced epithelial mesenchymal transition in human colon carcinoma cells," International Journal of Cancer, vol. 122, no. 8, pp. 1745-1756, 2008.

[15] S. K. Halder, R. D. Beauchamp, and P. K. Datta, "A specific inhibitor of TGF- $\beta$ receptor kinase, SB-431542, as a potent antitumor agent for human cancers," Neoplasia, vol. 7, no. 5, pp. 509-521, 2005.

[16] G. J. Inman, F. J. Nicolás, J. F. Callahan et al., "SB-431542 is a potent and specific inhibitor of transforming growth factor- $\beta$ superfamily type I activin receptor-like kinase (ALK) receptors ALK4, ALK5, and ALK7," Molecular Pharmacology, vol. 62, no. 1, pp. 65-74, 2002.

[17] S. Nakajima, R. Doi, E. Toyoda et al., "N-cadherin expression and epithelial-mesenchymal transition in pancreatic carcinoma," Clinical Cancer Research, vol. 10, no. 12, Part 1, pp. 41254133, 2004.

[18] S.-I. Shibata, H. Marushima, T. Asakurai et al., "Three-dimensional culture using a radial flow bioreactor induces matrix metalloprotease 7-mediated EMT-likeprocess in tumor cells via TGF $\beta 1$ /Smad pathway," International Journal of Oncology, vol. 34, no. 5, pp. 1433-1448, 2009.

[19] C. D. Morrison, J. G. Parvani, and W. P. Schiemann, “The relevance of the TGF- $\beta$ Paradox to EMT-MET programs," Cancer Letters, vol. 341, no. 1, pp. 30-40, 2013.

[20] R. Derynck, B. P. Muthusamy, and K. Y. Saeteurn, "Signaling pathway cooperation in TGF- $\beta$-induced epithelial-mesenchymal transition," Current Opinion in Cell Biology, vol. 31, pp. 5666, 2014. 


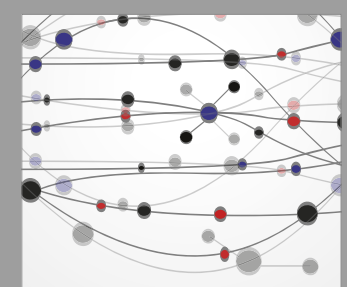

The Scientific World Journal
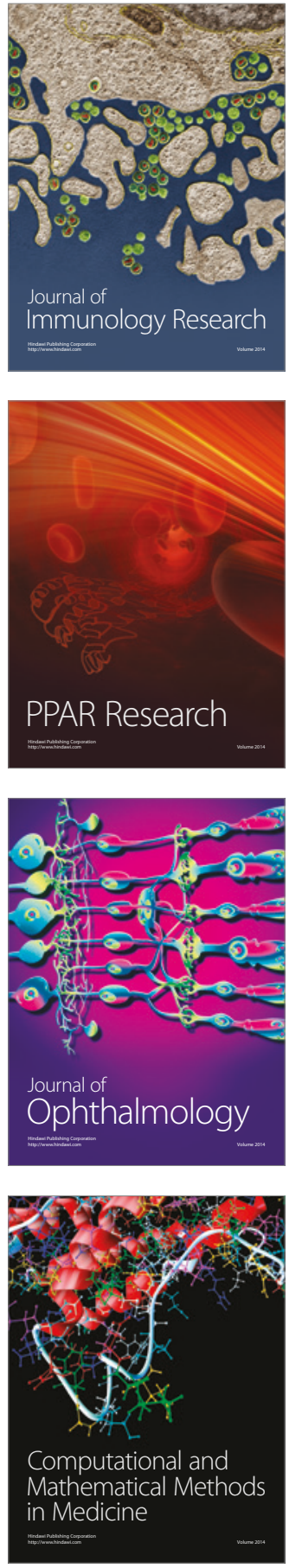

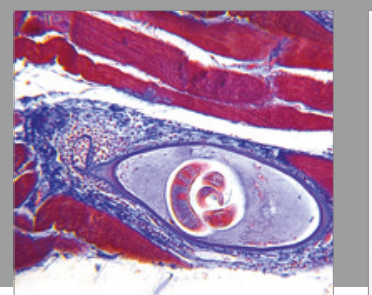

Gastroenterology Research and Practice
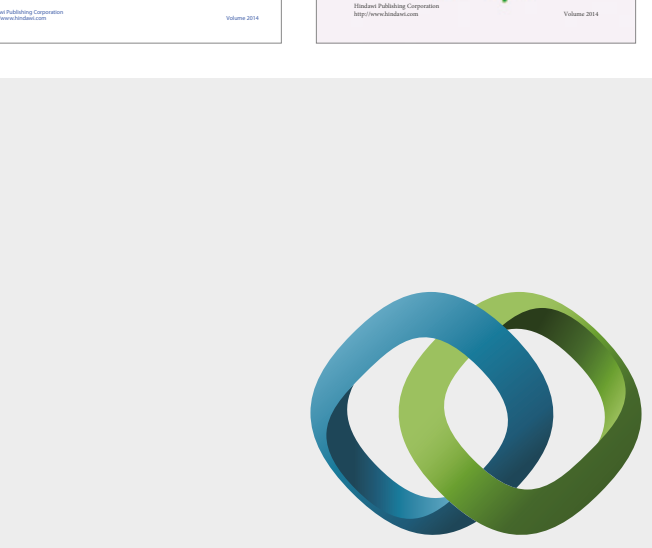

\section{Hindawi}

Submit your manuscripts at

https://www.hindawi.com
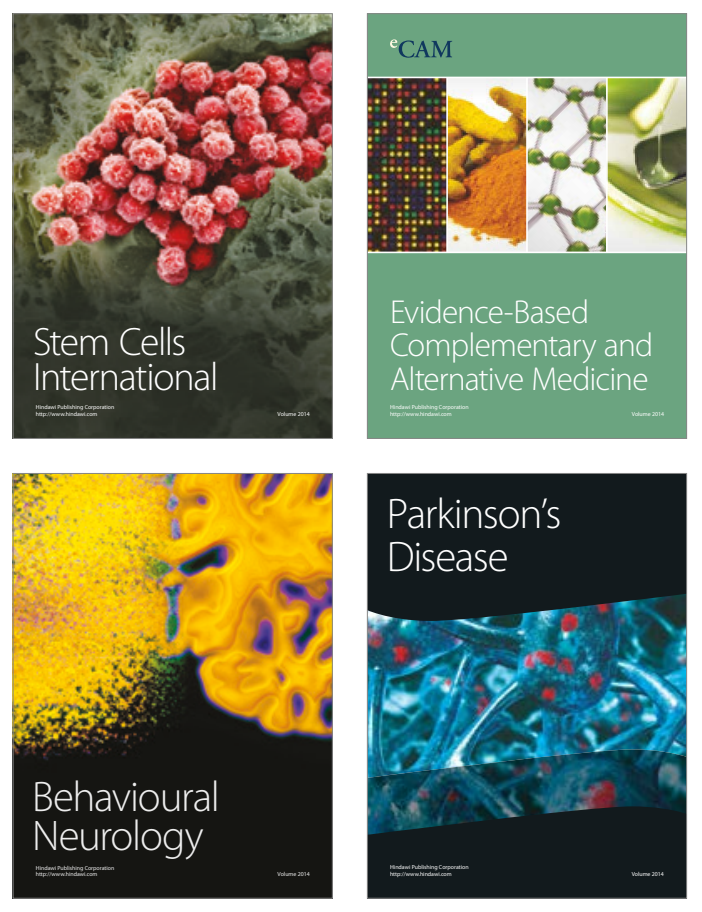
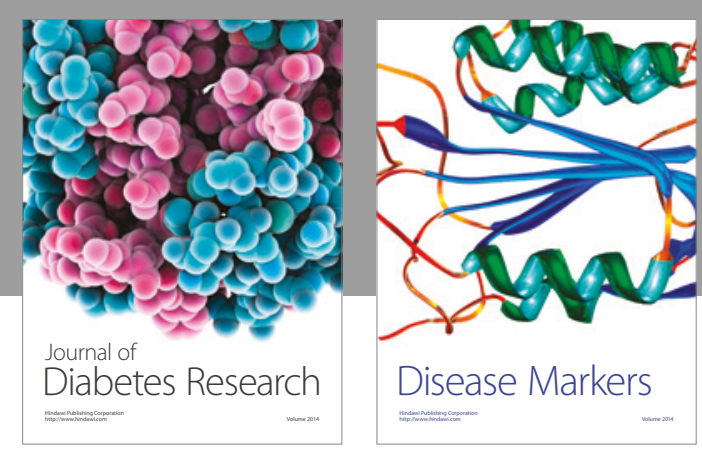

Disease Markers
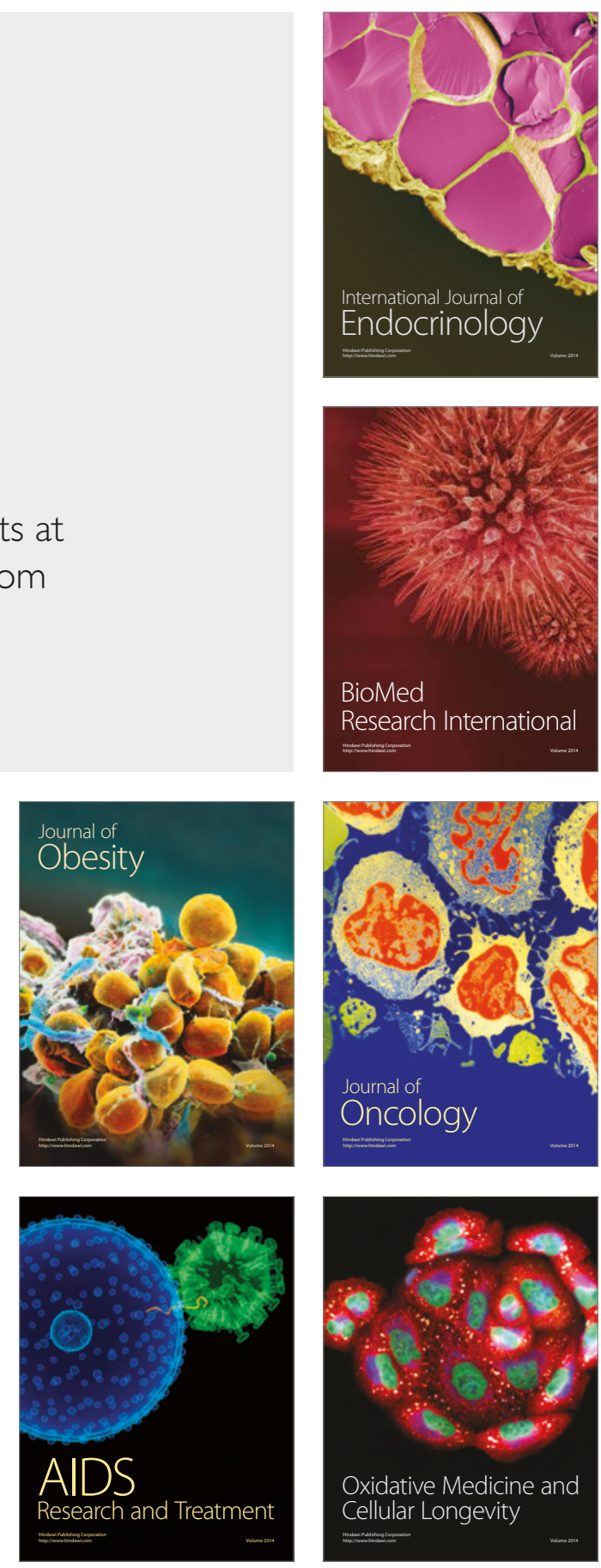